\title{
Interaction between gravitational waves and plasma waves in the Vlasov description
}

\author{
G. BRODIN, M. FORSBERG, \\ M. MARKLUND and D. ERIKSSON \\ Department of Physics, Umeå University, SE-901 87 Umeå, Sweden
}

(Received 29 October 2018)

\begin{abstract}
The nonlinear interaction between electromagnetic, electrostatic and gravitational waves in a Vlasov plasma is reconsidered. By using a orthonormal tetrad description the three-wave coupling coefficients are computed. Comparing with previous results, it is found that the present theory leads to algebraic expression that are much reduced, as compared to those computed using a coordinate frame formalism. Furthermore, here we calculate the back-reaction on the gravitational waves, and a simple energy conservation law is deduced in the limit of a cold plasma.
\end{abstract}

PACS: 04.30.Nk, 52.35.Bj, 95.30.Sf

\section{Introduction}

Much work has been devoted to special relativistic effects of plasmas [1], largely stimulated by the rapid progress in high power laser technology [2]. Several studies of plasmas in a general relativistic context have also been made, see .e.g. [3-5,11-13], considering for example plasmas in strongly curved space-times close to pulsars [14], multi-fluid plasma effects in general relativity [15-17], dynamo effects in strong gravity [18], the effect of two-temperature systems on scalar perturbations [19], viscous heating of accretion discs due to gravitational wave dissipation [20], or considering gravitational wave $(\mathrm{GW})$ propagation in a plasma medium, in particular nonlinear interactions in e.g. dusty plasmas [21,22] or MHD plasmas [23-27]. GW:s are currently opening up a promising new window for astronomy and astrophysics, e.g. astroseismology $[28,29]$, and the interaction between GW:s and electromagnetic fields have been proposed as a possible means to detect GW:s [30-34]. Moreover, the nonlinear interaction of a curved spacetime with and electromagnetic field can yield a multitude of interesting astrophysical and cosmological effects [35-38]. In a magnetized plasma, a basic effect is the linear coupling between electromagnetic (EM) waves and GW:s that occurs for propagation across a static magnetic field $[5] . \dagger$ This linear mechanism leads to the excitation of magnetohydrodynamic waves in a plasma [11-13]. Naturally linear coupling mechanisms is not sufficient if one is interested in the possibility of converting GW energy to frequencies different from that of the original source. Numerous examples of such mechanisms exists in

$\dagger$ For a discussion concerning the case of cosmological magnetic fields, see Refs. [6-10]. 
plasmas, involving e.g. frequency up-conversion due to nonlinear wave steepening [39],or various three-wave couplings between GWs and electromagnetic waves, e.g. $[40,41]$. Wave coupling mechanisms involving GWs are studied for several different reasons. In some cases, the emphasis is on the basic theory [42-45]. In other works, the focus is on GW detectors [46-48], on cosmology [49-51], or on astrophysical applications such as binary mergers [52], gamma ray bursts [53], pulsars [54] or supernovas [55].

In the present paper we will re-consider the problem of three-wave interacting between electrostatic (ES), electromagnetic (EM) and GW:s in a plasma, using a collisionfree kinetic description, i.e. the Vlasov equation [40]. In contrast to previous authors we will use a tetrad formalism [56], rather than a coordinate frame formalism, since the former formalism has been shown to significantly reduce the algebraic complexity. In particular, the coordinate frame formalism applied on the present problem produced very cumbersome algebraic expressions for the coupling coefficients [40], and as a result it was impossible to see if the growth rates for parametric processes where positive in general (when damping was omitted), which is related to the fulfillment of the so called Manley-Rowe relations [57]. The coefficients derived using the present tetrad formalism is shown to agree with previous works in the limit of a cold plasma [40]. For the case of a finite temperature, the coefficients found from the present formalism are algebraically much simpler than previous results. However, due to the complexity of the previously computed coefficients, a comparison cannot be easily made for the general case. Finally, in the present paper we also include the back-reaction on the GW, in contrast to previous works. This allows us to discuss the energy conservation properties, and an energy conservation law is presented in the low temperature limit.

\section{Basic equations in the tetrad formalism}

We consider the interaction between weak gravitational waves and a collisionless plasma in an external magnetic field. Since we consider nonempty space the background space-time is necessarily curved. However, if the wavelength of the gravitational waves and the interaction region is small relative to the background curvature we may take the background to be flat and static (the lowest order version of the high-frequency approximation), and consider the perturbed energy-momentum tensor corresponding to the perturbations of the electromagnetic and material fields.

For simplicity, the unperturbed plasma is assumed to be static, isotropic and homogeneous. Linearized, the Einstein field equations (EFE) take the form

$$
\square h_{a b}=-2 \kappa\left[\delta T_{a b}-\frac{1}{2} \delta T \eta_{a b}\right]
$$

provided the gauge condition $h^{a b}{ }_{, b}=0$ is fulfilled, which is equivalent to state that only tensorial perturbations are present. Here $\square \equiv\left[c^{-2} \partial_{t}^{2}-\partial_{z}^{2}\right], h_{a b}$ is the small deviation from the Minkowski background metric, i.e. $g_{a b}=\eta_{a b}+h_{a b}, \kappa \equiv 8 \pi G / c^{4}$, $\delta T_{a b}$ is the part of the energy-momentum tensor containing small electromagnetic and material field perturbations associated with the gravitational waves and $\delta T$ $=\delta T_{a}^{a}$. In the following it is understood that we neglect contributions of second order and higher in $h_{a b}$. In our notations $a, b, c, \ldots=0,1,2,3$ and $i, j, k, \ldots=1,2,3$ and the metric has the signature $(-+++)$.

In vacuum, a linearized gravitational wave can be transformed into the transverse 
and traceless (TT) gauge. Then we have the following line-element and corresponding orthonormal frame basis

$$
\begin{aligned}
\mathrm{d} s^{2}= & -c^{2} \mathrm{~d} t^{2}+\left(1+h_{+}(\xi)\right) \mathrm{d} x^{2}+\left(1-h_{+}(\xi)\right) \mathrm{d} y^{2} \\
& +2 h_{\times}(\xi) \mathrm{d} x \mathrm{~d} y+\mathrm{d} z^{2} \\
\mathbf{e}_{0} & \equiv c^{-1} \partial_{t}, \quad \mathbf{e}_{1} \equiv\left(1-\frac{1}{2} h_{+}\right) \partial_{x}-\frac{1}{2} h_{\times} \partial_{y} \\
\mathbf{e}_{2} & \equiv\left(1+\frac{1}{2} h_{+}\right) \partial_{y}-\frac{1}{2} h_{\times} \partial_{x}, \quad \mathbf{e}_{3} \equiv \partial_{z}
\end{aligned}
$$

where $\xi \equiv z-c t$ and $h_{+}, h_{\times} \ll 1$. As it turns out, the gravitational waves takes this form also in the particular case (propagation in an isotropic plasma) that we are considering. The difference to the vacuum case will be that $\xi=z-v_{\mathrm{ph}} t$, where $v_{\text {ph }}$ is the phase velocity of the gravitational wave. Note, however that the theory will be limited to the case $v_{\mathrm{ph}} \approx c$, due to the omittance of back-ground curvature effects. From now on we will refer to tetrad components rather than coordinate components.

We follow the covariant approach presented in [56]] for splitting the electromagnetic and material fields in a $1+3$ fashion. Suppose an observer moves with 4 velocity $u^{a}$. This observer will measure the electric and magnetic fields $E_{a} \equiv F_{a b} u^{b}$ and $B_{a} \equiv \frac{1}{2} \epsilon_{a b c} F^{b c}$, respectively, where $F_{a b}$ is the electromagnetic field tensor and $\epsilon_{a b c}$ is the volume element on hyper-surfaces orthogonal to $u^{a}$. It is convenient to introduce a 3 -vector notation $\mathbf{E} \equiv\left(E^{i}\right)=\left(E^{1}, E^{2}, E^{3}\right)$ etc. and $\nabla \equiv \mathbf{e}_{i}$. From now on we will assume that $u^{0}=c$ is the only nonzero component of $u^{a}$. As has been presented in e.g. Refs. [58,59]] the Maxwell equations contain terms coupling the electromagnetic field to the gravitational radiation field. Including terms that are linear in $h_{+}$and $h_{\times}$, but omitting terms that are quadratic and of higher order, Maxwell's equations are written as

$$
\begin{aligned}
\nabla \times \mathbf{B} & =\mu_{0}\left(\mathbf{j}+\mathbf{j}_{E}\right)+\frac{1}{c^{2}} \partial_{t} \mathbf{E}, \\
\partial_{t} \mathbf{B} & =\nabla \times \mathbf{E}-\mathbf{j}_{B}, \\
\nabla \cdot \mathbf{B} & =0 \\
\nabla \cdot \mathbf{E} & =\frac{\rho_{c}}{\varepsilon_{0}}
\end{aligned}
$$

where

$$
\begin{aligned}
\mathbf{j}_{E}= & -\frac{1}{2 c}\left[\left(E_{x}-c B_{y}\right) \dot{h}_{+}+\left(E_{y}+c B_{x}\right) \dot{h}_{\times}\right] \mathbf{e}_{1} \\
& +\frac{1}{2}\left[\left(E_{y}+c B_{x}\right) \dot{h}_{+}+\left(E_{x}-c B_{y}\right) \dot{h}_{\times}\right] \mathbf{e}_{2}, \\
\mathbf{j}_{B}= & -\frac{1}{2}\left[\left(E_{y}+c B_{x}\right) \dot{h}_{+}-\left(E_{x}-c B_{y}\right) \dot{h}_{\times}\right] \mathbf{e}_{1} \\
& -\frac{1}{2}\left[\left(E_{x}-c B_{y}\right) \dot{h}_{+}+\left(E_{y}+c B_{x}\right) \dot{h}_{\times}\right] \mathbf{e}_{2},
\end{aligned}
$$

are effective currents due to the GWs, see e.g. Ref. [45]], and the dot denotes derivatives with respect to the argument. The physical current- and charge-density are denoted $\mathbf{j}$ and $\rho_{c}$, respectively. Note that the absence of a GW source terms in Eqs. (2.7) and (2.8) is valid only within the given approximation, as can be seen from e.g. Ref. [45]], where source terms that are fully nonlinear in the GW-amplitude are included. In addition to the explicit source terms in (2.9)-(2.10), naturally the 
gravitational effects that is associated with the tetrad (2.3)-(2.4) must also be kept in mind.

Next we turn our attention to the particle description. The equation of motion for a particle of mass $m$ and charge $q$ in an electromagnetic and gravitational wave field is

$$
\frac{d}{d t} \mathbf{p}=q\left[\mathbf{E}+(\gamma m)^{-1} \mathbf{p} \times \mathbf{B}\right]-\mathbf{G}
$$

where $\gamma=\sqrt{1+p_{i} p^{i} /(m c)^{2}}$ and the four-momenta is $p^{a}=\gamma m d x^{a} / d t$. The gravitational force like term $G^{i} \equiv \Gamma_{a b}^{i} p^{a} p^{b} / \gamma m$, where $\Gamma_{a b}^{i}$ are the Ricci rotation coefficients, becomes

$$
\begin{aligned}
G_{1} & =\frac{1}{2}\left(v_{\mathrm{ph}}-p_{z} / \gamma m\right)\left[\dot{h}_{+} p_{1}+\dot{h}_{\times} p_{2}\right] \\
G_{2} & =\frac{1}{2}\left(v_{\mathrm{ph}}-p_{z} / \gamma m\right)\left[-\dot{h}_{+} p_{2}+\dot{h}_{\times} p_{1}\right] \\
G_{3} & =\frac{1}{2}(\gamma m)^{-1}\left[\dot{h}_{+}\left(p_{1}^{2}-p_{2}^{2}\right)+2 \dot{h}_{\times} p_{1} p_{2}\right]
\end{aligned}
$$

for weak gravitational waves propagating in the z-direction in Minkowski space, where $v_{\mathrm{ph}}$ is the phase velocity of the gravitational wave, which we at this point allow to deviate slightly from $c$.

Next we apply kinetic plasma theory, representing each particle species by a distribution function $f$ governed by the Vlasov equation. In tetrad form the Vlasov equation reads [59]]

$$
\mathcal{L} f=0
$$

where the Liouville operator is

$$
\mathcal{L} \equiv \partial_{t}+\left(c / p^{0}\right) p^{i} e_{i}+\left[F_{\mathrm{EM}}^{i}-\Gamma_{a b}^{i} p^{a} p^{b} c / p^{0}\right] \partial_{p^{i}}
$$

and the electromagnetic force responsible for geodesic deviation is $F_{\mathrm{EM}}^{i} \equiv q\left(E^{i}+\right.$ $\left.\epsilon^{i j k} p_{j} B_{k} / \gamma m\right)$. In vector notation the Vlasov equation reads

$$
\partial_{t} f+\frac{\mathbf{p} \cdot \nabla f}{\gamma m}+\left[q\left(\mathbf{E}+\frac{\mathbf{p} \times \mathbf{B}}{\gamma m}\right)-\mathbf{G}\right] \cdot \nabla_{\mathbf{p}} f=0
$$

where $\nabla_{\mathbf{p}} \equiv\left(\partial_{p_{1}}, \partial_{p_{2}}, \partial_{p_{3}}\right)$. In the absence of gravitational waves, the Vlasov equation has the following spatially homogeneous (thermodynamical) equilibrium solution, the Synge-Jüttner distribution, e.g. [40],

$$
f_{S J}=\frac{n_{0} \mu}{4 \pi(m c)^{3} K_{2}(\mu)} e^{-\mu \gamma}
$$

where $n_{0}$ is the spatial particle number density, $\mu \equiv m c^{2} / k_{B} T, k_{B}$ is the Boltzmann constant, $T$ the temperature and $K_{2}(\mu)$ is a modified Bessel function of second kind.

\section{Wave-wave interaction}

Next we let all quantities consist of a superposition of three waves of different kinds. Firstly we have a gravitational wave (frequency and wavevector $\left(\omega_{\mathrm{g}}, \mathbf{k}_{\mathrm{g}}\right)$ ), next an electromagnetic wave $\left(\omega_{\mathrm{em}}, \mathbf{k}_{\mathrm{em}}\right)$, and finally an electrostatic wave (Langmuir wave) $\left(\omega_{\mathrm{es}}, \mathbf{k}_{\mathrm{es}}\right)$. Since we are dealing with high-frequency waves, the mass and charge will in what follows refer to electrons. The three waves are assumed to obey the following matching conditions

$$
\omega_{\mathrm{g}}=\omega_{\mathrm{em}}+\omega_{\mathrm{es}}
$$


and

$$
\mathbf{k}_{\mathrm{g}}=k_{\mathrm{g}} \mathbf{e}_{3}=\mathbf{k}_{\mathrm{em}}+\mathbf{k}_{\mathrm{es}}
$$

Since there is no external magnetic field within our model, the gravitational wave does not induce an electric field at the frequency and wavevector $\left(\omega_{g}, \mathbf{k}_{g}\right)$. Furthermore, the metric perturbations at the combinations $\left(\omega_{1,2}, \mathbf{k}_{1,2}\right)$ can be neglected, as a consequence of the high-frequency approximation.

As a prerequisite to the nonlinear calculations we first consider linear theory. Linearizing the Vlasov equation, in the absence of EM-fields, the perturbed distribution function of the GW is given by.

$$
f_{\mathrm{g}}=\frac{2 i p_{a} G_{a}}{\hat{\omega}_{\mathrm{g}}} \frac{\partial f_{S J}}{\partial|p|^{2}}
$$

where we have introduced the notation $\hat{\omega}_{\mathrm{g}}=\omega_{\mathrm{g}}-k_{\mathrm{g}} c p_{3} / p_{0}$. Noting that the different GW-polarizations obey

$$
\left(\omega_{\mathrm{g}}^{2}-k_{\mathrm{g}}^{2} c^{2}\right) h_{\times}=-2 \kappa \delta T_{12}=-2 \kappa \int \frac{p_{1} p_{2}}{m \gamma} f_{\mathrm{g}} d^{3} p
$$

and

$$
\left(\omega_{\mathrm{g}}^{2}-k_{\mathrm{g}}^{2} c^{2}\right) h_{+}=-2 \kappa\left(\delta T_{11}-\delta T_{22}\right)=-2 \kappa \int \frac{\left(p_{1}^{2}-p_{2}^{2}\right)}{m \gamma} f_{\mathrm{g}} d^{3} p
$$

we deduce the same dispersion relation for both GW-polarizations, namely

$$
D_{g}\left(\omega_{\mathrm{g}}, k_{\mathrm{g}}\right)=\omega_{\mathrm{g}}^{2}-k_{\mathrm{g}}^{2} c^{2}-2 \kappa n_{0} \int \frac{p_{1}^{2} p_{2}^{2}}{m \gamma} \frac{\partial f_{S J}}{\partial|p|^{2}} d^{3} p=0
$$

where we here and from now on use the normalization $\int f_{S J} d^{3} p=1$ of the unperturbed distribution. In the high-frequency approximation, the last term of (3.6) is a small correction, comparable to the contribution from the background curvature [5]], and thus we may use the the approximation $D_{g}=\omega_{\mathrm{g}}^{2}-k_{\mathrm{g}}^{2} c^{2}$. Similarly, from the linearized Vlasov equation (without gravitational fields), together with Maxwell's equations we deduce the dispersion relation for electromagnetic waves

$$
D_{\mathrm{em}}\left(\omega_{\mathrm{em}}, k_{\mathrm{em}}\right)=1-\frac{k_{\mathrm{em}}^{2} c^{2}}{\omega_{\mathrm{em}}^{2}}+\frac{\omega_{p}^{2}}{\omega_{\mathrm{em}}} \int \frac{2 p_{2}^{2}}{\gamma \hat{\omega}_{\mathrm{em}}} \frac{\partial f_{S J}}{\partial|p|^{2}} d^{3} p=0
$$

and for electrostatic waves

$$
D_{\mathrm{es}}\left(\omega_{\mathrm{es}}, k_{\mathrm{es}}\right)=1-\frac{2 m \omega_{p}^{2}}{k_{\mathrm{es}}^{2}} \int \frac{\mathbf{k}_{\mathrm{es}} \cdot \mathbf{p}}{\gamma \hat{\omega}_{\mathrm{es}}} \frac{\partial f_{S J}}{\partial|p|^{2}} d^{3} p=0
$$

where $\omega_{p}=\left(n_{0} q^{2} / \varepsilon_{0} m\right)^{1 / 2}$ is the electron plasma frequency. When nonlinear interactions are taken into account, the wave amplitudes will be time-dependent. We note that as far as the linear terms are concerned the only modification needed is the simple substitution $D_{\mathrm{es}} E_{\mathrm{es}}=\left(\partial D_{\mathrm{es}} / \partial \omega_{\mathrm{es}}\right) \partial \tilde{E}_{\mathrm{es}} / \partial t$, where the tilde denotes the weakly time-dependent amplitude, and similarly for the other waves [57]]. Next, for definiteness, we assume the wave-vectors to span the plane perpendicular to $\mathbf{e}_{2}$. Furthermore, we note that for symmetry reasons, the $h_{\times}$-polarization in this geometry couples to the EM-wave polarized with the electric field along $\mathbf{e}_{2}$ (nonlinearly combined with the electrostatic wave), whereas the $h_{+}$-polarization couples to the EM-waves with magnetic field along $\mathbf{e}_{2}$. These two cases are similar, and from now 
6G. BRODIN, M. FORSBERG, M. MARKLUND and D. ERIKSSON

on we limit ourselves to the former choice of polarization. The amplitude evolution for $\tilde{h}_{\times}$is found by keeping resonant terms proportional to both the EM and electrostatic wave in the energy-momentum tensor. Next we use Eq. (3.4), generalized to keep the nonlinear terms and a weakly time-dependent amplitude. Substituting the expression for $f_{g}$ from the Vlasov equation, but now with second order nonlinear terms included, we obtain

$$
\omega_{\mathrm{g}} \frac{\partial \tilde{h}_{\times}}{\partial t}=2 \varepsilon_{0} \kappa C_{\mathrm{g}} \tilde{E}_{\mathrm{es}} \tilde{E}_{\mathrm{em}}
$$

where the coupling coefficient $C_{\mathrm{g}}$ is

$$
\begin{aligned}
C_{\mathrm{g}}= & {\left[\frac{\mathbf{k}_{\mathrm{es}} \cdot \mathbf{e}_{1}}{k_{\mathrm{es}}}+\frac{\omega_{p}^{2}}{k_{\mathrm{es}}} \int \frac{p_{1} p_{2}^{2}}{\gamma \hat{\omega}_{\mathrm{em}} \hat{\omega}_{\mathrm{es}}}\right.} \\
& \left.\times\left\{2 \frac{\mathbf{k}_{\mathrm{em}} \cdot \mathbf{k}_{\mathrm{es}}}{\hat{\omega}_{\mathrm{g}} \gamma m}\left[\frac{\hat{\omega}_{\mathrm{em}} \omega_{\mathrm{es}}}{\omega_{\mathrm{em}} \hat{\omega}_{\mathrm{es}}}+\frac{\hat{\omega}_{\mathrm{es}}}{\hat{\omega}_{\mathrm{em}}}\right] \frac{\partial f_{S J}}{\partial|p|^{2}}+4 \mathbf{p} \cdot \mathbf{k}_{\mathrm{es}} \frac{\partial^{2} f_{S J}}{\partial\left(|p|^{2}\right)^{2}}\right\} d^{3} p .\right]
\end{aligned}
$$

To find the EM-wave evolution, we now include all resonant nonlinear source terms for the EM-wave (effective gravitational currents, nonlinear terms involving the gravitational force, and nonlinearities coming directly from the tetrad), and solve for $\partial \tilde{E}_{\mathrm{em}} / \partial t$, in which case we obtain

$$
\frac{\partial D_{\mathrm{em}}}{\partial \omega_{\mathrm{em}}} \frac{\partial \tilde{E}_{\mathrm{em}}}{\partial t}=-C_{\mathrm{em}} \tilde{h}_{\times} \tilde{E}_{\mathrm{es}}^{*}
$$

where

$$
\begin{aligned}
& C_{\mathrm{em}}=\left(-\frac{k_{\mathrm{em}}^{2} c^{2}}{2 \omega_{\mathrm{em}}^{2}}-\frac{\omega_{\mathrm{g}}}{2 \omega_{\mathrm{em}}}\right) \frac{\mathbf{k}_{e s} \cdot \mathbf{e}_{1}}{k_{e s}}+\frac{\omega_{p}^{2}}{\omega_{\mathrm{em}} k_{e s}} \int \frac{p_{2}^{2}}{\gamma \hat{\omega}_{\mathrm{em}} \hat{\omega}_{\mathrm{es}}} \\
& \times\left\{\mathbf{k}_{\mathrm{es}} \cdot \mathbf{e}_{1}\left(2 \frac{\omega_{\mathrm{g}} \hat{\omega}_{\mathrm{es}}}{\hat{\omega}_{\mathrm{g}}}-\frac{\hat{\omega}_{\mathrm{g}} \omega_{\mathrm{es}}}{\hat{\omega}_{\mathrm{es}}}\right) \frac{\partial f_{S J}}{\partial|p|^{2}}-\frac{4 p_{1} \omega_{\mathrm{g}} \hat{\omega}_{\mathrm{em}}}{\hat{\omega}_{\mathrm{g}}} \mathbf{p} \cdot \mathbf{k}_{\mathrm{es}} \frac{\partial^{2} f_{S J}}{\partial\left(|p|^{2}\right)^{2}}\right\} d^{3} p .
\end{aligned}
$$

Finally the calculation is completed by doing analog calculation for the electrostatic wave evolution, and the result is

$$
\frac{\partial D_{\mathrm{es}}}{\partial \omega_{\mathrm{es}}} \frac{\partial \tilde{E}_{\mathrm{es}}}{\partial t}=-C_{\mathrm{es}} \tilde{h}_{\times} \tilde{E}_{\mathrm{em}}^{*}
$$

where

$$
\begin{array}{r}
C_{\mathrm{es}}=\frac{\omega_{p}^{2}}{k_{\mathrm{es}}}\left[\frac{\mathbf{k}_{\mathrm{em}} \cdot \mathbf{e}_{1}}{2 \omega_{p}^{2}}-\int \frac{p_{2}^{2}}{\gamma \hat{\omega}_{\mathrm{es}}}\left(\frac{\mathbf{k}_{\mathrm{em}} \cdot \mathbf{e}_{1}}{\hat{\omega}_{\mathrm{em}}}-\frac{2 \mathbf{k}_{\mathrm{es}} \cdot \mathbf{k}_{\mathrm{em}} \omega_{\mathrm{g}} p_{1}}{\gamma \hat{\omega}_{\mathrm{es}} \hat{\omega}_{\mathrm{g}} \omega_{\mathrm{em}} m_{e}}\right.\right. \\
\left.\left.-\frac{\left(\omega_{\mathrm{g}}-p_{3} k_{\mathrm{g}} / \gamma m_{e}\right) \mathbf{k}_{\mathrm{es}} \cdot \mathbf{e}_{1}+\left(2 p_{1} / \gamma m_{e}\right) \mathbf{k}_{\mathrm{es}} \cdot \mathbf{k}_{\mathrm{g}}}{\hat{\omega}_{\mathrm{em}} \hat{\omega}_{\mathrm{es}}}\right) \frac{\partial f_{S J}}{\partial|p|^{2}} d^{3} p\right]
\end{array}
$$

The coupling coefficients $C_{\mathrm{es}}$ and $C_{\mathrm{em}}$ (although not $C_{\mathrm{g}}$ ) has been calculated in Ref. [40]], using a coordinate frame formalism. As can be seen the previously computed coefficients are algebraically much more complicated, and thus a comparison is difficult in general. For the special case where the temperature of $f_{S J}$ approaches zero, the coefficients simplify a lot, and we obtain

$$
C_{\mathrm{es}}=C_{\mathrm{em}}=\frac{\mathbf{k}_{\mathrm{es}} \cdot \mathbf{e}_{1} \omega_{\mathrm{g}}}{k_{\mathrm{es}} \omega_{\mathrm{em}}}
$$


which agrees with Ref. [40]]. Furthermore, we find that in our case that also the gravitational case coefficient is similar, and we can define the common coupling coefficient $C \equiv C_{\mathrm{es}}=C_{\mathrm{em}}=C_{\mathrm{g}}$. As a result of the agreement of the coefficients, the energy change of each of the waves in the cold limit can be written

$$
\begin{aligned}
& \frac{d W_{\mathrm{g}}}{d t}=-\omega_{\mathrm{g}} V \\
& \frac{d W_{\mathrm{es}}}{d t}=\omega_{\mathrm{es}} V
\end{aligned}
$$

and

$$
\frac{d W_{\mathrm{em}}}{d t}=\omega_{\mathrm{em}} V
$$

where the energy density of each wave is

$$
\begin{gathered}
W_{\mathrm{g}}=\omega_{\mathrm{g}}^{2}\left|\tilde{h}_{\times}\right|^{2} / 2 \kappa, \\
W_{\mathrm{es}}=\varepsilon_{0} \omega_{\mathrm{es}}\left(\partial D_{\mathrm{es}} / \partial \omega_{\mathrm{es}}\right)\left|\tilde{E}_{\mathrm{es}}\right|^{2}, \\
W_{\mathrm{em}}=\varepsilon_{0} \omega_{\mathrm{em}}\left(\partial D_{\mathrm{em}} / \partial \omega_{\mathrm{em}}\right)\left|\tilde{E}_{\mathrm{em}}\right|^{2}
\end{gathered}
$$

and

$$
V=\varepsilon_{0} C \tilde{h}_{\times} \tilde{E}_{\mathrm{em}}^{*} \tilde{E}_{\mathrm{es}}^{*}+\text { c.c }
$$

where c.c. denotes complex conjugate. The equations (3.16)-(3.18) together with (3.1) thus shows that the three-wave interaction process conserves the total waveenergy.

\section{Summary and conclusion}

In the present paper we have reconsidered the process of three-wave interaction between gravitational-, electromagnetic- and electrostatic waves in a collisionfree plasma. Using an ortonormal tetrad description, the algebraic complexity of the coupling coefficients is much reduced, as compared to previous authors [40]]. Two of the coupling coefficients (for the electrostatic and the electromagnetic wave) can be shown to agree with previous results in the cold limit. The third coefficient (for the GW) has not been calculated before. Inclusion of the back-reaction on the GW makes it possible to deduce and energy conservation law (Eqs (3.16)-(3.18) together with (3.1)) in the cold limit. The energy conservation law is a natural consequence of the Manley-Rowe relations [57]], which makes the same coefficient appear in all three coupled equations ( $c . f . C \equiv C_{\mathrm{es}}=C_{\mathrm{em}}=C_{\mathrm{g}}$.). However, this relation was only verified in the cold limit. Although the present coefficients were not extremely complicated, it has proved to be difficult to deduce whether the coupling coefficients are symmetric (i.e. fulfilling the Manley-Rowe relations), for the general case of a finite temperature. An interesting question, that remains for future research, is thus to decide whether this is due to some principal lack of a canonical Hamiltonian structure (that is the basic source of symmetric coupling coefficients, see e.g. [60]]) of the Einstein-Maxwell-Vlasov system, or if the difficulties are merely due to the algebraical complexity.

\section{Acknowledgements}

This research was partially supported by the Swedish Research Council and the Swedish Graduate School of Space Technology. 
8G. BRODIN, M. FORSBERG, M. MARKLUND and D. ERIKSSON

\section{References}

[1] P. K. Shukla, N. N. Rao, M. Y. Yu, and N. L. Tsintsadze, Phys. Reports 138, 1 (1986).

[2] M. Marklund and P. K. Shukla, Rev. Mod. Phys. 78, 591 (2006)

[3] J. Daniel and T. Tajima, Phys. Rev. D, 55, 5193 (1997).

[4] M. Marklund, G. Brodin and P. K. Shukla, Phys. Scripta T82, 130, (1999).

[5] L. P. Grishchuk and A. G. Polnarev, General Relativity and Gravitation Vol. 2 ed. A. Held (Plenum Press, New York, 1980) pp. 416-430.

[6] C.G. Tsagas, P.K.S. Dunsby, and M. Marklund, Phys. Lett. B 561, 17 (2003).

[7] G. Betschart, C. Zunckel, P. Dunsby, and M. Marklund, Phys. Rev. D 72, $123514(2005)$.

[8] C. Zunckel, G. Betschart, P. Dunsby, and M. Marklund, Phys. Rev. D 73, 103509 (2006).

[9] C. G. Tsagas, Phys. Rev. D 75, 087901 (2007).

[10] G. Betschart, C. Zunckel, P. K. S. Dunsby, and M. Marklund, Phys. Rev. D 75, 087902 (2007).

[11] D. Papadopoulos et al., A \& A 377, 701 (2001).

[12] J. Moortgat and J. Kuijpers, A \& A 402, 905 (2003).

[13] J. Moortgat and J. Kuijpers, Phys. Rev. D, 70, 023001 (2004).

[14] K.S. Thorne, D.A. MacDonald, and R.H. Price, The Membrane Paradigm (Yale University Press, 1986).

[15] M. Marklund, P.K.S. Dunsby, G. Betschart, M. Servin, and C.G. Tsagas, Class. Quantum Grav. 20, 1823 (2003).

[16] G. Betschart, P.K.S. Dunsby, and M. Marklund, Class. Quantum Grav. 21, 2115 (2004).

[17] M.A. Rahman and M.H. Ali, Gen. Rel. Grav., DOI: 10.1007/s10714-0090891-x (2009).

[18] M. Marklund and C.A. Clarkson, MNRAS 358, 892 (2005).

[19] J. Moortgat and M. Marklund, MNRAS 369, 1813 (2006).

[20] B. Kocsis and A. Loeb, Phys. Rev. Lett. 101, 041101 (2008).

[21] G. Brodin, M. Marklund, and P.K. Shukla, JETP Lett. 81, 135 (2005).

[22] M. Forsberg, G. Brodin, M. Marklund, P.K. Shukla, and J. Moortgat, Phys. Rev. D 74, 064014 (2006).

[23] A. Källberg, G. Brodin, and M. Bradley, Phys. Rev. D 70, 044014 (2004).

[24] M.D. Duez, Y.T. Liu, S.L. Shapiro, and B.C. Stephens, Phys. Rev. D 72, $024028(2005)$

[25] M.D. Duez, Y.T. Liu, S.L. Shapiro, and B.C. Stephens, Phys. Rev. D 72, $024029(2005)$

[26] H. Isliker, I. Sandberg, and L. Vlahos, Phys. Rev. D 74, 104009 (2006).

[27] B.D. Farris, T.K. Li, Y.T. Liu, and S.L. Shapiro, Phys. Rev. D 78, 024023 (2008).

[28] N. Andersson and K.D. Kokkotas, MNRAS 299, 1059 (1998).

[29] N. Andersson, K. Glampedakis, and L. Samuelsson, MNRAS 396, 894 (2009).

[30] V.B. Braginskii, L.P. Grishchuk, A.G. Doroshkevich, Ia.B. Zeldovich, I.D. Novikov, and M.V. Sazhin, Soviet Physics JETP 38, 865 (1974).

[31] F. Pegoraro, E. Picasso, and L.A. Radicati, J. Phys. A: Math. Gen. 11, 1949 (1978).

[32] C.M. Caves, Phys. Lett. B 80, 323 (1979). 
[33] G. Brodin and M. Marklund, Class. Quantum Grav. 20, L45 (2003).

[34] G. Stedman, R. Hurst, and K. Schreiber, Opt. Comm. 279, 124 (2007).

[35] C. Tsagas, Class. Quantum Grav. 22, 393 (2005).

[36] M. Giovannini, Class. Quantum Grav. 23, R1 (2006).

[37] J.D. Barrow, R. Maartens, and C.G. Tsagas, Phys. Rep. 449, 131 (2007).

[38] E. Fenu and R. Durrer, Phys. Rev. D 79, 024021 (2009).

[39] M. Forsberg and G. Brodin, Phys. Rev. D 77, 024050 (2008).

[40] G. Brodin and M. Marklund, Phys. Rev. Lett. 82. 3012, (1999).

[41] M. Servin, G.Brodin, M. Bradley and M. Marklund, Phys. Rev. E, 62, 8493 (2000).

[42] M. Servin and G. Brodin, Phys. Rev. D 68, 044017 (2003).

[43] J. T. Mendonça, Plasma Phys. Control. Fus., 44, B225, (2002).

[44] A. B. Balakin et al., J. Math. Phys., 44, 5120 (2003)

[45] G. Brodin, M. Marklund and P.K.S. Dunsby, Phys. Rev. D 62, 104008 (2000).

[46] F.Y. Li., M. X. Tang., Int. J. Mod. Phys. D, 11, 1049 (2002)

[47] G. Brodin and M. Marklund, Class. Quantum Grav. 20, 45 (2003).

[48] R. Ballantini et al., Class. Quantum Grav. 20, 3505 (2003).

[49] D. Papadopoulos, Class Quantum Grav. 19, 2939 (2002).

[50] M. Marklund, P.K.S. Dunsby and G. Brodin, Phys. Rev. D 62, 101501(R) (2000).

[51] P. A. Hogan and E. M. O'Shea, Phys. Rev D 65, 124017 (2002).

[52] G. Brodin, M. Marklund and M. Servin, Phys. Rev. D 63, 124003 (2001).

[53] L. Vlahos et al., Astrophys. J., 604, 297 (2004).

[54] H. J. M. Cuesta, Phys. Rev. D 65, 64009 (2002).

[55] G. Brodin, M. Marklund and P. K. Shukla, JETP Lett. 81, 135 (2005) 135; Pisma Zh.Eksp.Teor.Fiz. 81, 169 (2005).

[56] G. F. R. Ellis and H. van Elst, Cosmological models, Theoretical and Observational Cosmology, ed. M Lachi'eze-Rey (Dordrecht: Kluwer) (1999).

[57] J. Weiland and H. Wilhelmsson, Coherent Nonlinear Interaction of Waves in Plasmas, (Pergamon press New York, 1977).

[58] M. Marklund, G. Brodin, and P.K.S. Dunsby, Astrophys. J. 536, 875 (2000).

[59] M. Servin, G. Brodin and M. Marklund, Phys. Rev. D 64, 024013 (2001).

[60] J. Larsson, J. Plasma Phys., 69, 211 (2003). 